University of Louisville

ThinkIR: The University of Louisville's Institutional Repository

Faculty Scholarship

3-2011

\title{
The thick disk in the galaxy NGC 4244 from S4G imaging.
}

\author{
Sebastien Comeron \\ Korea Astronomy and Space Science Institute \\ Johan H. Knapen \\ Universidad de La Laguna \\ Kartik Sheth \\ National Radio Astronomy Observatory \\ Michael Regan \\ Space Telescope Science Institute \\ Joannah L. Hinz \\ University of Arizona \\ See next page for additional authors
}

Follow this and additional works at: https://ir.library.louisville.edu/faculty

Part of the Astrophysics and Astronomy Commons

\section{Original Publication Information \\ Comeron, Sebastien, et al. "The Thick Disk in the Galaxy NGC 4244 from S4G Imaging." 2011. The Astrophysical Journal 729(1): 12 pp.}

This Article is brought to you for free and open access by ThinkIR: The University of Louisville's Institutional Repository. It has been accepted for inclusion in Faculty Scholarship by an authorized administrator of ThinkIR: The University of Louisville's Institutional Repository. For more information, please contact thinkir@louisville.edu. 


\section{Authors}

Sebastien Comeron, Johan H. Knapen, Kartik Sheth, Michael Regan, Joannah L. Hinz, Armando Gil de Paz, Karin Menendez-Delmestre, Juan Carlos Munoz-Mateos, Mark Seibert, Taehyun Kim, E. Athanassoula,

Albert Bosma, Ronald J. Buta, Bruce G. Elmegreen, Luis C. Ho, Benne W. Holwerda, Eija Laurikainen, Heikki Salo, and Eva Schinnerer 


\title{
THE THICK DISK IN THE GALAXY NGC 4244 FROM S ${ }^{4}$ G IMAGING
}

\author{
Sébastien Comerón $^{1,2}$, Johan H. Knapen ${ }^{2,3}$, Kartik Sheth ${ }^{4,5,6}$, Michael W. Regan $^{7}$, Joannah L. Hinz ${ }^{8}$, \\ Armando Gil de Paz ${ }^{9}$, Karín Menéndez-Delmestre ${ }^{10}$, Juan-Carlos Muñoz-Mateos ${ }^{9}$, Mark Seibert $^{10}$, Taehyun Kim ${ }^{4,11}$, \\ E. Athanassoula ${ }^{12}$, Albert Bosma ${ }^{12}$, Ronald J. Buta ${ }^{13}$, Bruce G. Elmegreen ${ }^{14}$, Luis C. Ho ${ }^{10}$, Benne W. Holwerda $^{15}$, \\ Eija LauriKainen ${ }^{16,17}$, HeikKi Salo ${ }^{16}$, AND Eva SchinNerer ${ }^{18}$ \\ ${ }^{1}$ Korea Astronomy and Space Science Institute, 61-1 Hwaam-dong, Yuseong-gu, Daejeon 305-348, Republic of Korea \\ ${ }^{2}$ Instituto de Astrofísica de Canarias, E-38200 La Laguna, Spain \\ ${ }^{3}$ Departamento de Astrofísica, Universidad de La Laguna, E-38205 La Laguna, Tenerife, Spain \\ ${ }^{4}$ National Radio Astronomy Observatory/NAASC, 520 Edgemont Road, Charlottesville, VA 22903, USA \\ ${ }^{5}$ Spitzer Science Center, 1200 East California Boulevard, Pasadena, CA 91125 \\ ${ }^{6}$ California Institute of Technology, 1200 East California Boulevard, Pasadena, CA 91125, USA \\ ${ }^{7}$ Space Telescope Science Institute, 3700 San Martin Drive, Baltimore, MD 21218, USA \\ ${ }^{8}$ Steward Observatory, University of Arizona, 933 North Cherry Avenue, Tucson, AZ 85721, USA \\ ${ }^{9}$ Departamento de Astrofísica, Universidad Complutense de Madrid, 28040 Madrid, Spain \\ 10 The Observatories of the Carnegie Institution of Washington, 813 Santa Barbara Street, Pasadena, CA 91101, USA \\ ${ }^{11}$ Astronomy Program, Department of Physics and Astronomy, Seoul National University, Seoul 151-742, Republic of Korea \\ ${ }^{12}$ Laboratoire d'Astrophysique de Marseille (LAM), UMR6110, CNRS/Université de Provence/CNRS, Technopôle de Marseille Étoile, \\ 38 rue Frédéric Joliot Curie, 13388 Marseille Cédex 20, France \\ ${ }^{13}$ Department of Physics and Astronomy, University of Alabama, Box 870324, Tuscaloosa, AL 35487, USA \\ ${ }^{14}$ IBM T. J. Watson Research Center, 1101 Kitchawan Road, Yorktown Heights, NY 10598, USA \\ ${ }^{15}$ Astrophysics, Cosmology, and Gravity Centre (ACGC), Astronomy Department, University of Cape Town, Private Bag X3, \\ 7700 Rondebosch, Republic of South Africa \\ ${ }^{16}$ Astronomy Division, Department of Physical Sciences, FIN-90014 University of Oulu, P.O. Box 3000, Oulu, Finland \\ ${ }^{17}$ Finnish Centre of Astronomy with ESO (FINCA), University of Turku, Väisäläntie 20, FI-21500, Piikkiö, Finland \\ ${ }^{18}$ Max-Planck-Institut für Astronomie, Königstuhl 17, D-69117 Heidelberg, Germany \\ Received 2010 August 16; accepted 2010 November 29; published 2011 February 7
}

\begin{abstract}
If thick disks are ubiquitous and a natural product of disk galaxy formation and/or evolution processes, all undisturbed galaxies that have evolved during a significant fraction of a Hubble time should have a thick disk. The late-type spiral galaxy NGC 4244 has been reported as the only nearby edge-on galaxy without a confirmed thick disk. Using data from the Spitzer Survey of Stellar Structure in Galaxies $\left(\mathrm{S}^{4} \mathrm{G}\right)$ we have identified signs of two disk components in this galaxy. The asymmetries between the light profiles on both sides of the mid-plane of NGC 4244 can be explained by a combination of the galaxy not being perfectly edge-on and a certain degree of opacity of the thin disk. We argue that the subtlety of the thick disk is a consequence of either a limited secular evolution in NGC 4244, a small fraction of stellar material in the fragments which built the galaxy, or a high amount of gaseous accretion after the formation of the galaxy.
\end{abstract}

Key words: galaxies: individual (NGC 4244) - galaxies: photometry - galaxies: spiral - galaxies: structure

Online-only material: color figures

\section{INTRODUCTION}

Thick disks are detected in edge-on and very inclined galaxies as an excess of flux at some distance from the mid-plane, typically at a few thin disk scale heights. They usually have exponential profiles with a larger scale height than the "canonical" or thin disks. Thick disks were first detected by Tsikoudi (1979) and were defined by Burstein (1979). A thick disk has subsequently been found in the Milky Way (Gilmore \& Reid 1983) and in basically all kinds of disk galaxies (see the introduction of Yoachim \& Dalcanton 2008 and references therein).

Several theories of thick disk formation have been proposed. It has been suggested that thick disks are formed through mechanisms of internal evolution, namely, disk heating by gravitational instabilities (Bournaud et al. 2007, 2009) and/or stellar scattering by encounters with features such as dark matter subhalos, spiral arms, or giant molecular clouds (Villumsen 1985; Hänninen \& Flynn 2002; Benson et al. 2004; Hayashi \& Chiba 2006; Haywood 2008; Kazantzidis et al. 2008; Schönrich \& Binney 2009a, 2009b; Bournaud et al. 2009). In this picture, the thick disk formation may take less than $1 \mathrm{Gyr}$ in the case of fast dynamical heating by a very clumpy initial disk (Bournaud et al. 2009) or may be much slower (secular evolution; Villumsen 1985; Hänninen \& Flynn 2002; Benson et al. 2004; Kazantzidis et al. 2008). The specific type of secular evolution which causes a slow heating of the stellar orbits has been termed "blurring" by Schönrich \& Binney (2009a, 2009b).

Another possibility is that the thick disk is formed through accretion of stellar material from merging satellites at the time of galaxy formation (Abadi et al. 2003; Yoachim \& Dalcanton 2006). A third possibility is that the thick disk is a consequence of in situ star formation (Brook et al. 2004; Elmegreen \& Elmegreen 2006) or of star formation with a high initial velocity dispersion in very massive star clusters (Kroupa 2002).

Whatever the thick disk formation mechanism is, it has to act over most, if not all, disk galaxies as thick disks have been found to be ubiquitous (Dalcanton \& Bernstein 2002; Yoachim $\&$ Dalcanton 2006). Therefore, if any disk galaxy was found not to possess a thick disk, it would likely have suffered a peculiar origin and/or evolution. A search in the literature yields a candidate, NGC 4244, which has been claimed either to be a doubtful case (Yoachim \& Dalcanton 2006) or not to have a 
thick disk at all (Fry et al. 1999). NGC 4244 is a highly inclined apparently undisturbed Scd galaxy located 4.4 Mpc away (Seth et al. 2005a). At that distance, one arcsecond is equivalent to roughly $21 \mathrm{pc}$ in linear scale. NGC 4244 is nearly bulgeless, except for a compact and bright nuclear star cluster (see Seth et al. 2008 for a detailed study on the nuclear cluster; the bulgeto-disk ratio is 0.03 according to Fry et al. 1999). NGC 4244 exhibits systematic displacements of the mid-plane of a few tens of parsecs with a wavelength of a couple of kiloparsecs (this phenomenon has been termed "corrugation" and in the case of NGC 4244 it has been studied by Florido et al. 1991). Kodaira \& Yamashita (1996) found NGC 4244 to have less warm dust and star formation than galaxies of the same Hubble type and Hi content, thus labeling it as an "anemic" galaxy. Finally, de Jong et al. (2007) found a sharp disk cutoff which they suggest to have a dynamical origin. Roskar et al. (2008) suggest this cut-off to be related to the radius at which a drop in the star formation rate is found. This drop is suggested to be associated to a drop in the cooled gas surface density. NGC 4244 could be weakly interacting with NGC 4214, which has a similar recession velocity and is found at a projected distance of $120 \mathrm{kpc}$ from NGC 4244.

Several studies have attempted to identify the structural components of NGC 4244. Bergstrom et al. (1992) found no evidence for a massive stellar halo. Fry et al. (1999) detected only a thin disk. Hoopes et al. (1999) found that, unlike some other edge-on galaxies, NGC 4244 has its ionized gas concentrated in the thin disk. Strickland et al. (2004) found no evidence for a radio, UV, or X-ray emitting halo. Seth et al. (2005b) found that the scale heights of the older stellar components are larger than those of the younger components, which could be an indicator of the existence of a thick disk; however, the difference in scale heights between the mainsequence population and the red giant branch (RGB) population is smaller than in all the other galaxies studied in their paper. They also detected what could be interpreted as a halo. Tikhonov \& Galazutdinova (2005) claimed to have discovered a tenuous extended halo with a scale length of several kiloparsecs in NGC 4244. Seth et al. (2007) found a faint halo component $2.5 \mathrm{kpc}$ above the mid-plane at the position of the minor axis.

To sum up, previous studies on NGC 4244 show little to no emission at any wavelength outside its well-known thin disk. As nearly all very inclined disk galaxies have been shown to host a thick disk, the absence of it in NGC 4244, if confirmed, would be an indication of a peculiar evolution or formation mechanism of this galaxy.

In this paper, we present results of a study of NGC 4244 based on a Spitzer Survey of Stellar Structure in Galaxies $\left(S^{4} \mathrm{G}\right)$ $3.6 \mu \mathrm{m}$ image (Sheth et al. 2010; Section 2), confirming that this galaxy does in fact have signs of two disk components. For this purpose, we have fitted the galaxy using GALFIT (version 3.0; Peng et al. 2002; Peng et al. 2010; Section 3) and produced luminosity profiles of NGC 4244 at different galactocentric radii (Section 4). We describe the luminosity profiles and compare them with a simple inclined two-disk model with a diffuse dust contribution in the thin disk in Section 5. We present a summary of the NGC 4244 main structural components in Section 6 and discuss our findings in Section 7.

\section{PROCESSING OF THE $\mathrm{S}^{4} \mathrm{G} 3.6 \mu \mathrm{m}$ IMAGE OF NGC 4244}

The $S^{4} \mathrm{G}$, described by Sheth et al. (2010), is a survey using the $3.6 \mu \mathrm{m}$ and $4.5 \mu \mathrm{m}$ filters of the Spitzer Space Telescope Infrared Array Camera (Fazio et al. 2004) with the aim of studying the stellar mass distribution in a sample of 2331 local galaxies.

The $3.6 \mu \mathrm{m}$ image of NGC 4244 we have used for our study has been processed by the $S^{4} G$ pipelines, resulting in a mosaicked flat-fielded image and in a mask of foreground stars, background galaxies, and bad pixels (Sheth et al. 2010). However, as we aim to reach the faintest possible surface brightness level, we have manually refined the mask and closely examined the background. The background of the image is at a level of $0.0330 \mathrm{mJy} \mathrm{sr}^{-1}$ (corresponding to $\mu_{3.6 \mu \mathrm{m}}(A B)=$ $24.2 \mathrm{mag} \mathrm{arcsec}^{-2}$ ). The $\sigma$ of the background is of the order of $0.002 \mathrm{mJy} \mathrm{sr}^{-1}$, which is comparable to the offsets applied to the individual frames in order to obtain a mosaic with a uniform background $\left(0.0015 \mathrm{mJy} \mathrm{str}^{-1}\right)$. We find a systematic background gradient which is shown in the top left panel of Figure 1. This gradient has an amplitude which is approximately two times larger than that of the $\sigma$ of the background. The gradient is unlikely to be caused by zodiacal light or by the concentration of bright stars at the northern part of the frame because it is not consistent with that of the $S^{4} \mathrm{G} 4.5 \mu \mathrm{m}$ image which was obtained for the same galaxy. We have modeled the background using a third-order two-dimensional polynomial fit to all unmasked pixels at a distance of 250 pixels (187".5) or more from the galaxy mid-plane. In this procedure, there is no risk of contamination by any component of the galaxy because we are going farther than any trace of extended component found by Tikhonov \& Galazutdinova (2005) using the RGB star-counting method and because the surface brightness of the halo detected by Seth et al. (2007) drops below the $S^{4} G$ limiting magnitude at that distance from the mid-plane. We then subtracted the modeled background from the original image (the result is shown in the top right panel of Figure 1).

We rotated the image 43.6 counterclockwise in order to place the mid-plane of the galaxy horizontally (lower panel of Figure 1). We estimate that the uncertainty in the rotation angle is about $1^{\circ}$. The rotation angle has been calculated by finding the mid-plane for all galactocentric distances $r<350$ arcsec $(r<7.5 \mathrm{kpc})$. This rotation angle is within the uncertainties consistent with the value of $42^{\circ}$ used by Olling (1996) and Fry et al. (1999). Once the galaxy has been rotated, we can define as the top part of the galaxy the area extending above its mid-plane and as the bottom part of the galaxy the region below it. This definition is arbitrary, but it will be useful because the galaxy is not symmetric with respect to its mid-plane.

NGC 4244 has a warp-like feature starting from galactocentric radii of $4^{\prime}-5^{\prime}$ in both optical (indicated with an arrow in Figure 2; Sloan Digital Sky Survey Data Release 7 (SDSS DR7) image; Abazajian et al. 2009) and near-infrared bands (indicated with arrows in the bottom panel of Figure 1) in agreement with the warp properties measured by Saha et al. (2009). The color image of the galaxy (top panel of Figure 2) shows that this warp-like feature is delineated by a succession of blue knots $\left[\left(g_{\text {knot }}-r_{\text {knot }}\right)-\left(g_{\text {surroundings }}-r_{\text {surroundings }}\right) \sim-0.4\right]$, which are areas of ongoing star formation as confirmed by the $\mathrm{H} \alpha$ image of Kennicutt et al. (2008). This indicates that the warp-like feature is likely to be a product of the presence of spiral arm fragments embedded in a very inclined galaxy disk (this possibility has been discussed by van der Kruit 1979). In the bottom panel of Figure 2, the warp-like feature is delineated by the deviations of the tips of the gray areas (indicated with "A" arrows) from the horizontal orientation. This warp-like feature is part of the corrugation pattern described by Florido et al. (1991), as outside the "warp," at a lower surface brightness, the disk mid-plane bends 

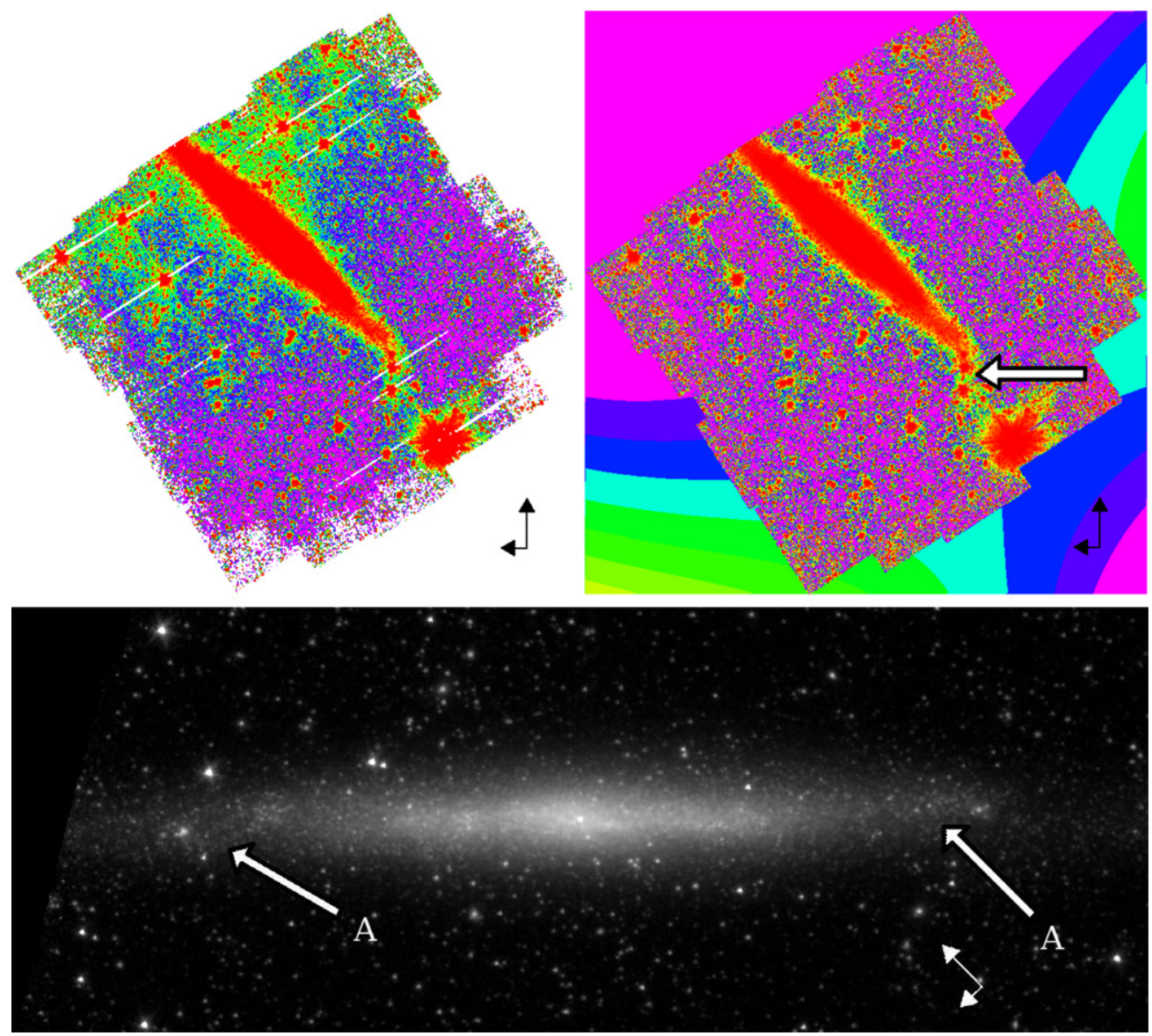

Figure 1. Top panels: $3.6 \mu \mathrm{m} \mathrm{S}{ }^{4} \mathrm{G}$ image of NGC 4244 before (left) and after (right) removing the gradient in the background. We have used the same histogram equalization display for both images. These images have been smoothed using a Gaussian function with $\sigma=1.25$ pixels. North is up and east is left. Lower panel: $3.6 \mu \mathrm{m} \mathrm{S}{ }^{4} \mathrm{G}$ image of NGC 4244 rotated 43.6 counterclockwise in such a way that the galaxy plane is horizontal. In this frame north is roughly located at the top left and east at the bottom left. The image is $15^{\prime}$ or $19.2 \mathrm{kpc}$ in length. The distance between the nuclear cluster and the "A" arrows is around $5^{\prime}$. The display for this panel is logarithmic. The arrow in the top right panel indicates a light streak caused by the combination of the light of three foreground stars. The " $\mathrm{A}$ " arrows in the bottom panel indicate the location of the warp-like feature.

(A color version of this figure is available in the online journal.)

in the other sense (as shown in Figure 2). In the top images of Figure 1 (in which the color-scale distribution is based on a histogram equalization algorithm), the warp-like feature we have described may be confused with a light streak which goes downward from the southeastern edge of NGC 4244 (opposite to the warp-like feature and indicated by an arrow). This streak is caused by three relatively bright aligned stars whose pointspread functions overlap in the histogram equalized display.

The warp-like distortion we find coincides with that seen in the $R$ band by Fry et al. (1999). These authors state that the optical "warp" they see is coincident with the H I warp discussed by Olling (1996), but this conclusion is due to them having used an image of NGC 4244 flipped along the mid-plane. The warp found in $\mathrm{H}$ I by Olling (1996) starts at a galactocentric radius of $r \sim 10^{\prime}$, which would place it at larger galactocentric radii than shown in the image in the bottom panel of Figure 1.

An inspection of the SDSS DR7 image of NGC 4244 (Figure 2; indicated by the arrow labeled with letter "B") shows a dust lane parallel to the mid-plane and slightly above it. This indicates that what we have defined to be the bottom part of the galaxy is facing us and thus we can describe it as the near side of the galaxy. This was already pointed out by Olling (1996) who found that the inclination of the galaxy is $i=84.5$. The dust lane is undetectable in the $\mathrm{S}^{4} \mathrm{G}$ images, even in a $3.6-4.5 \mu \mathrm{m}$ color-index map.

\section{GALFIT ANALYSIS OF NGC 4244}

In order to know whether a single-disk description is good enough to account for the structural components of NGC 4244, we have run Galfit (version 3.0; Peng et al. 2002; Peng et al. 2010) on the $S^{4} G$ NGC 4244 image using the GALFIDL procedures and display routines (see http://sun3.oulu. $\mathrm{fi} / \sim \mathrm{hsalo} /$ galfidl.html). GALFIT is a code designed for fitting one or more two-dimensional components to an image.

As an input we used the background-subtracted $\mathrm{S}^{4} \mathrm{G}$ image, the refined mask, and a "sigma" image, which accounts for the statistical uncertainties associated with each pixel. The "sigma" image is made using GALFIDL, which takes into account the pixel weights varying in different parts of the image mosaic.

\subsection{Inclined Disk Model}

In this model, GALFIT assumes a radially decaying exponential profile. In order to run it, we fixed the center of the galaxy at the position of the nuclear cluster and the disk position angle (P.A.) as 46.4 which corresponds to $90^{\circ}$ in the rotated images we present in Figure 3. We left as free parameters the disk luminosity, the disk axis ratio, and the disk scale length. As the galaxy appears to contain no bulge, no further components are a priori needed. The major caveat of modeling a very inclined galaxy in this manner is that the inclined disk GALFIT model is an 

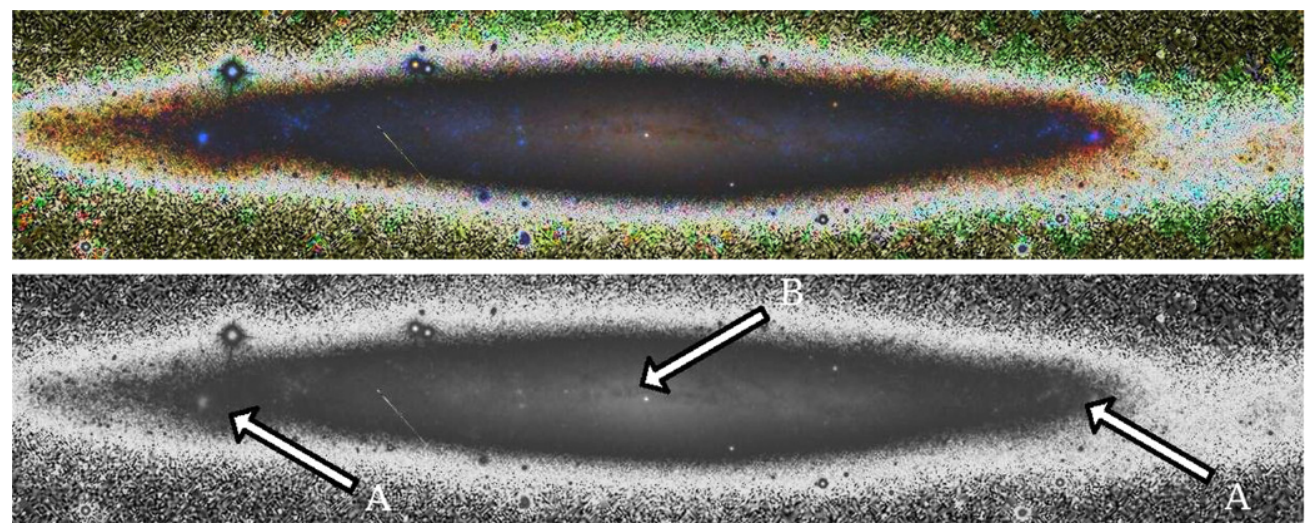

Figure 2. SDSS DR7 ugriz image of NGC 4244 oriented identically and at the same scale as in the bottom panel of Figure 1 . The top panel contains a color version of the image and the bottom panel has a black and white version of it. In the color image, the blue knots denote star formation and the reddish colors dusty areas. In the bottom panel, the "A" arrows indicate the location of the warp-like feature which is described in Section 2. The "B" arrow indicates a dust lane that runs parallel to the mid-plane of the galaxy and is located slightly above it.

(A color version of this figure is available in the online journal.)

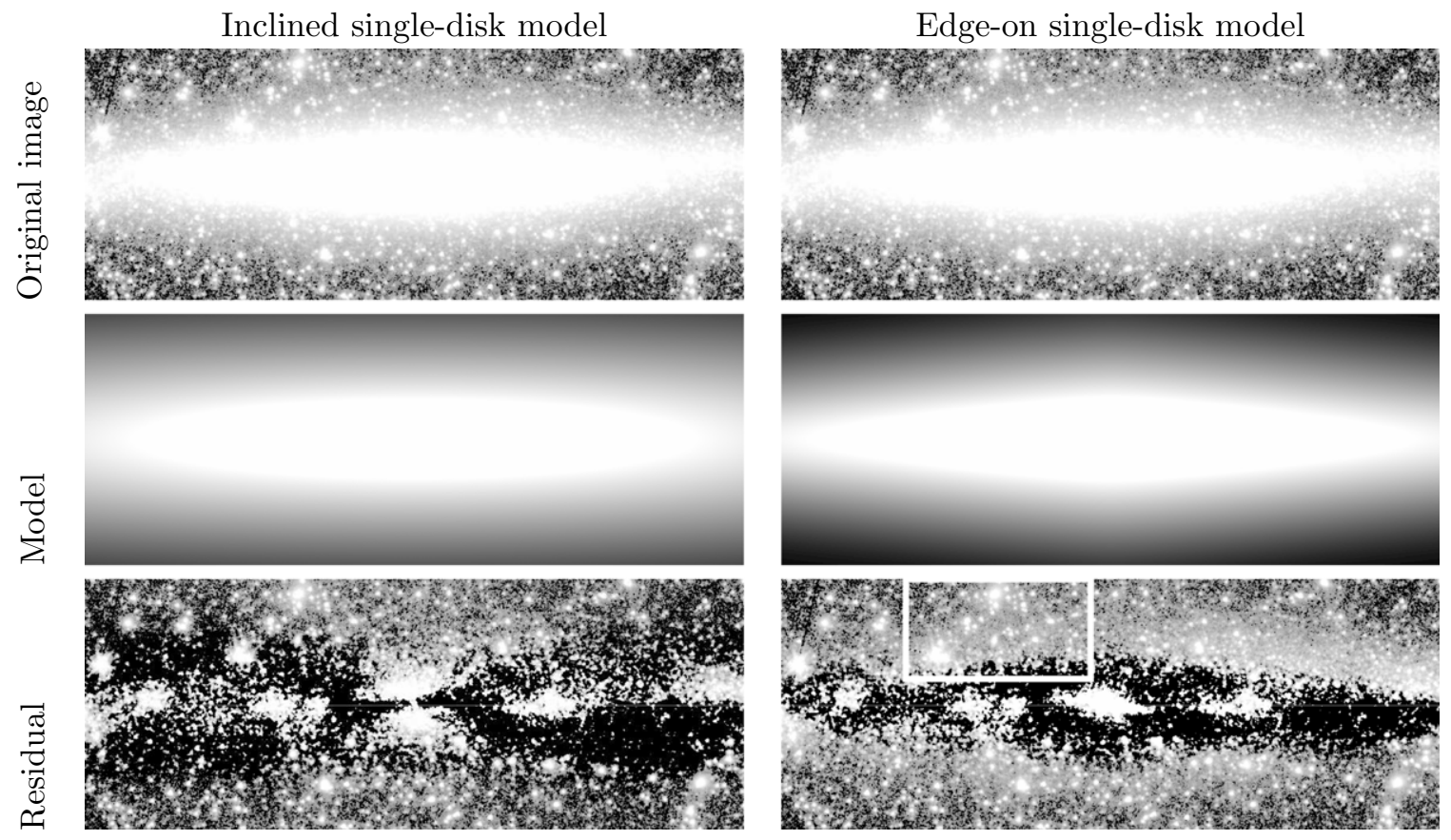

Figure 3. Inclined single-disk model is presented on the left and the edge-on single-disk model is presented on the right. From top to bottom, the images correspond to the original $\mathrm{S}^{4} \mathrm{G}$ background-subtracted frame, to the GALFIT model, and to the residual image. All the images are in a logarithmic display. The white rectangle indicates an arc-like feature that we interpret as possibly of tidal origin.

infinitely thin circular disk model, which will cause all the vertical extension of the galaxy to be interpreted as caused by the disk inclination. The resulting disk has an axis ratio $d / D=0.14$, which gives a disk inclination of $i=82^{\circ}$. Considering that in our model the disk is infinitely thin, which will automatically cause the disk to be less inclined than it really is, this value is compatible with the inclination of $i=84.5$ given by Olling (1996). The inclined disk model and the residual image (the original background-subtracted image from which the model has been subtracted) can be seen in the left column of Figure 3 .

\subsection{Edge-on Disk Model}

When applying the edge-on disk GALFIT model we are assuming that the inclination angle of the galaxy is $i=90^{\circ}$. The function which GalfiT uses is

$$
\Sigma(r, z)=\Sigma_{0} \frac{|r|}{h_{\mathrm{r}}} K_{1}\left(\frac{|r|}{h_{\mathrm{r}}}\right) \operatorname{sech}^{2}\left(\frac{z}{h_{\mathrm{z}}}\right),
$$

where $\Sigma_{0}$ is the central surface brightness, $K_{1}$ is a Bessel function, $r$ is the galactocentric distance, $h_{\mathrm{r}}$ is the major axis disk scale length, $z$ is the distance above the plane, and $h_{z}$ is the scale length perpendicular to the plane, as developed by van der Kruit \& Searle (1981) for the light distribution in edge-on disks. As for the other model, we fixed the center of the galaxy at the position of the nuclear cluster and the disk P.A. as $90^{\circ}$. We left as free parameters the disk luminosity, the disk scale length, and the disk scale height. The major caveat of modeling a not fully edge-on galaxy in this manner is that we are ignoring the fact that part of the apparent scale height is an effect of the galaxy inclination. The edge-on disk model and the residual image can be seen in the right column of Figure 3.

\subsection{Comparison of the Two NGC 4244 GaLFIT Models}

The main difference between the inclined and the edge-on model (see Figure 3 ) is that in the first case the galaxy model 


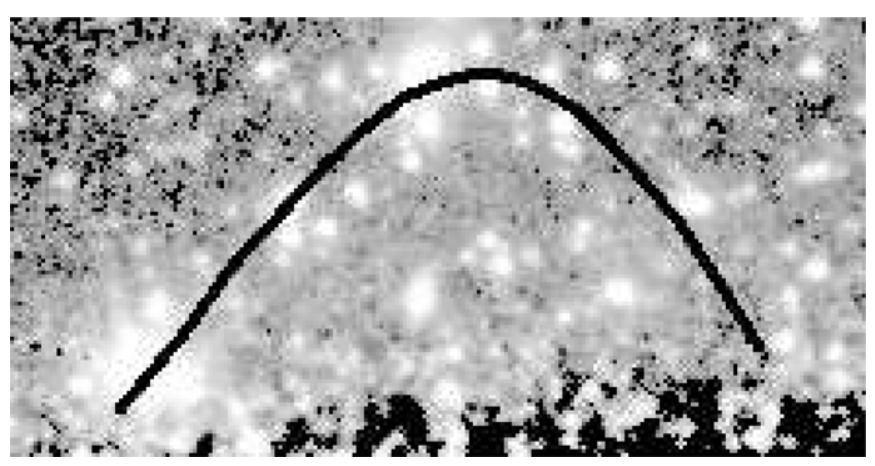

Figure 4. Close-up of the box in the lower right panel in Figure 3. The proposed path of the arc-like feature is indicated with a black line.

isophotes are elliptical and that in the second case they are diamond-shaped. The reason for this is that, in the first case, we are seeing the projection of an infinitely thin disk, which gives as a result a very eccentric ellipse. In the second case, we are seeing a diamond shape because the GALFIT light distribution for an edge-on disk has a cusp at the galactocentric distance $r=0$. The real galaxy image is more peaked than the inclined disk model for galactocentric distances larger than $r=4^{\prime}$ (which causes the butterfly-shaped dark areas in the residual image) but has rounder isophotes than the edge-on model near $r=0$. The residual image shows that, again, the inclined disk model is inaccurate at high galactocentric radii where it predicts a light distribution parallel to the $z$-axis much wider than observed.

In both models, the residual image shows bright patches close to the mid-plane. In the residuals of the edge-on model, these patches are reminiscent of a small bar or/and a spiral arm system seen nearly edge-on. The residual image of the edge-on model also shows an envelope of residual light which may be related to a thick disk. The extension of this envelope roughly corresponds to that of the galaxy's white-color envelope in the color panel in Figure 2. However, part of this residual emission, especially that at the top right side of the image, has more to do with the warp-like feature described in Section 2 than with the presence of a thick disk, as indicated in Figure 2 by the behavior of blue knots of star formation at high galactocentric radii.

An arc-like feature is visible in the residual images of both models at the top-left side of the frame and at a very low surface brightness (Figures 3 and 4). It is more visible in the edge-on model residual image (indicated with a white rectangle) and we speculate that it could be related to some kind of tidal feature, as its shape is somewhat similar to that observed in other very inclined galaxies (such as in NGC 5907 studied by Shang et al. 1998 and Martínez-Delgado et al. 2008 and in NGC 4013 by Martínez-Delgado et al. 2009; these cases are admittedly much more spectacular than that described here). This feature is knotty, which can be explained by the presence of globular clusters or star-forming regions in a tidally disrupted galaxy.

Neither of the models we ran reflect accurately the behavior of the stellar components in NGC 4244. However, the simple modeling we have done is enough to show that a simple singlecomponent description is not adequate to describe NGC 4244's structural properties.

\section{VERTICAL LUMINOSITY PROFILES}

In order to search for traces of a thick disk, we have produced luminosity profiles parallel to the $z$-axis of the galaxy at different galactocentric radii $(r)$. These luminosity profiles are shown in Figure 5.

To produce the luminosity profiles, we first made vertical bins with a width of $225^{\prime \prime} 0$ (300 pixels or $4.8 \mathrm{kpc}$ ) and centered at galactocentric distances $r=-337^{\prime \prime} .5,-225^{\prime \prime} .0,-112^{\prime \prime} .5,0^{\prime \prime} 0$, $112^{\prime \prime} .5,225^{\prime \prime} .0$, and $337^{\prime \prime} .5$. The $225^{\prime \prime} .0$ bin width has been chosen because it offers a good compromise between spatial resolution and the profile's signal to noise. As the bin width is double the separation between bins, the bins are overlapping. For each bin we determined the surface brightness at a given $z$ making a median of the counts excluding the pixels which were masked. We determined the location of the mid-plane of the galaxy from the profiles obtained in each bin. This was done by folding the profiles and minimizing the difference between the upper and lower halves of the light profile, with a precision of 0.1 pixel. For each bin we made a visual guess of where the galaxy effectively ends. The noise level was calculated by producing the standard deviation of those pixels at higher $z$. This is a conservative estimate of the noise, as the profile is produced by doing the median of the pixels in the bin. We smoothed the profiles in the vertical direction in order to get a higher signal at low surfacebrightness areas. This smoothing has been done logarithmically and using a vertical scale height of 5 pixels (3".75).

As the near and the far side of the galaxy are different, we have fitted them separately. We decided to make exponential fits at ranges in $z$ where the data appear roughly exponential. The fit was made in magnitude units and all the points were given equal weight. We fitted one or two exponentials to each profile depending on whether we detected - by eye - any trace of a thick disk or not. The fitting ranges appear in Table 1, and they are indicated in Figures 5 and 6. When preparing the fits, we considered all the luminosity values above the noise level to be reliable. The noise in our profiles is $\mu_{3.6 \mu \mathrm{m}}(A B) \sim$ $26.5 \mathrm{mag} \operatorname{arcsec}^{-2}$. If we had not taken the background gradient into account, as described in Section 2, this level would have been $\mu_{3.6 \mu \mathrm{m}}(A B) \sim 26.0 \mathrm{mag} \operatorname{arcsec}^{-2}$. The results of the fits are overlaid on the profiles in Figure 5 and are summarized in Table 1.

\section{RESULTS}

We find that all but one of the far side profiles can be fitted with a single exponential. Only for the profiles at $-225^{\prime \prime} .0 \leqslant$ $r \leqslant 112.5$ there is an extra faint component which cannot be fitted by an exponential. The near side profiles are well fitted by two exponential components except for that at $r=337^{\prime \prime} .5$ (Figure 5). The scale heights of all these fits are roughly stable with varying galactocentric radius.

\subsection{Looking for a Thick Disk in the Luminosity Profiles}

The luminosity profiles (Figure 5) expose a lack of symmetry between the far and near sides of NGC 4244. Once an inner exponential has been fitted, it is clearly seen that larger $z$ sections of the near side profiles can be fitted with a shallower exponential. By comparing lower and upper profiles, we can see that the concave inflection points between two exponentials in the near side profiles (which are especially noticeable for $-112^{\prime \prime} .5,112^{\prime \prime} .5,225^{\prime \prime} .0$, and $337^{\prime \prime} .5$ ) have no clear counterpart in the far side part of the galaxy. The inflection point is not so evident in the near side profile at $r=-337^{\prime \prime} .5$ and $r=-225^{\prime \prime} .0$ and it is not noticeable at all for $r=337 . .5$ may be due to some effect related to this region is being affected by the warp-like feature. The inflection point is also subtle at $r=0^{\prime} .0$. 

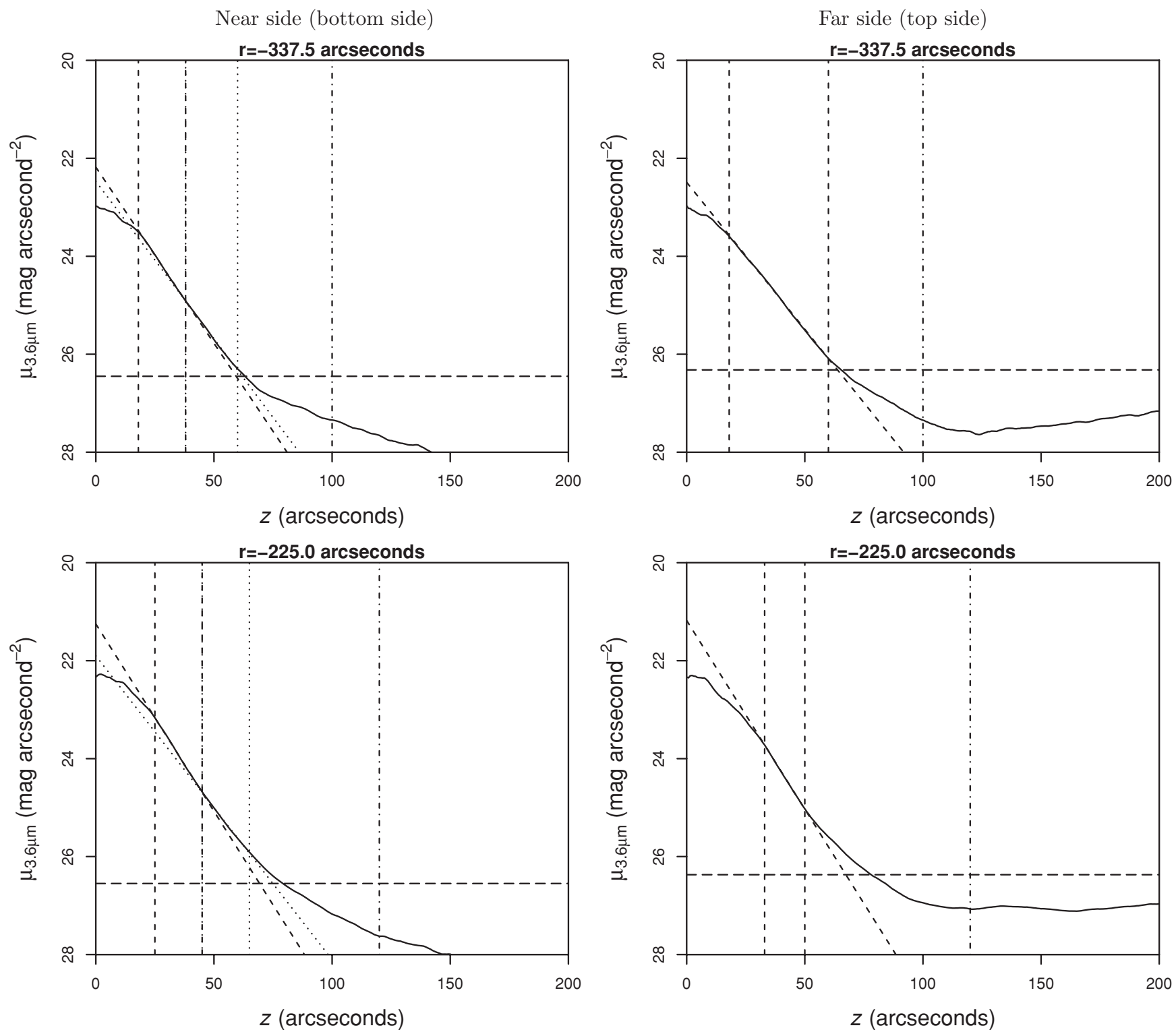

Figure 5. Luminosity profiles along the $z$-axis at different galactocentric radii (seen in the subplot's titles). Short dashed lines indicate the fits to inner exponentials and the range of $z$ at which the fit has been done (vertical lines). Dotted lines indicate the fits to outer exponentials and the range of $z$ at which the fit has been done (vertical lines) for these profiles for which an outer exponential has been fitted (in some cases the vertical line indicating the inner boundary of the outer exponential fitting range is coincident with the outer boundary of the inner exponential fitting range). Dash-dotted lines indicate the minimum $z$ at which the noise level has been calculated. The horizontal long-dashed line indicates the noise level. The profiles from the near side of the galaxy are on the left-hand side and those from the far side are on the right-hand side. The error bar length at the noise level is on the order of the line width.

An excess of light appears in the far side of the galaxy, for $-225^{\prime \prime} .0 \leqslant r \leqslant 112^{\prime \prime} .5$, but it cannot be easily fitted by an exponential and, even where we could in principle have done it, the fit would have to be done over a much smaller range in $z$. This excess corresponds to the arc-like feature in the residual image obtained from GALFIT (indicated with a white rectangle in the bottom right panel in Figure 3 and shown in Figure 4). As it has a surface brightness comparable to that of the second exponential we find in the near side profiles, it could hide part of a thick disk in the far side of the galaxy for radii from $r=-225^{\prime \prime} .0$ to $r=112$ ".5. However, the lack of traces of a thick disk in the far side profiles, even at galactocentric radii not affected by the non-exponential excess of light, indicates that a thick disk there is less obvious than in the near side of the galaxy, if it is present at all.

The luminosity profiles show some anti-symmetry, which is expected for a more or less symmetric warp or a symmetric spiral arm pattern embedded in the thin disk. This anti-symmetry is seen in Figure 5 whose panels at large negative radius at the near side resemble most the panels at large positive radius at the far side and vice versa.

The scale height of both disk components is stable from $r=-337^{\prime \prime} .5$ to $r=112^{\prime \prime} .5$ (Figure 7). For this range of galactocentric radii, the thin disk scale height is on average $16.22(350 \mathrm{pc})$ for the far side of the galaxy and 14". 1 (300 pc) for the near side of it. At the same range of galactocentric radii the thick disk has a scale height of $17^{\prime \prime} .4$ (370 pc). For comparison Seth et al. (2007) fitted a single disk with $\sim 300 \mathrm{pc}$ in scale height. At galactocentric radii larger than $r=112^{\prime \prime} 5$, both the thin and thick disks apparently flare (this can also be seen at the right side of Figure 2 and has been described by van der Kruit \& Searle 1981). This apparent flare is related to the warp-like feature, which we argued may be related to the spiral structure of the disk. 

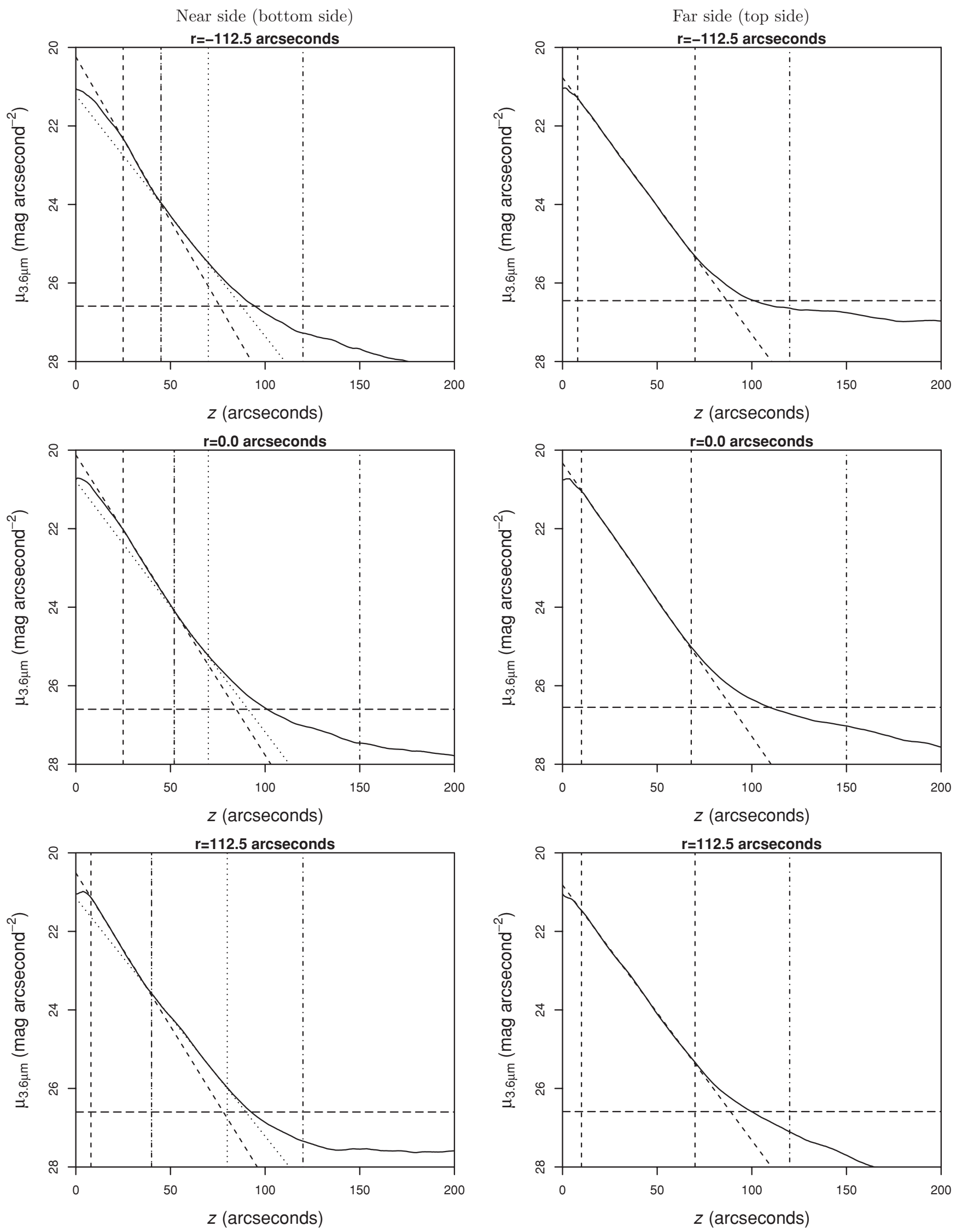

Figure 5. (Continued) 

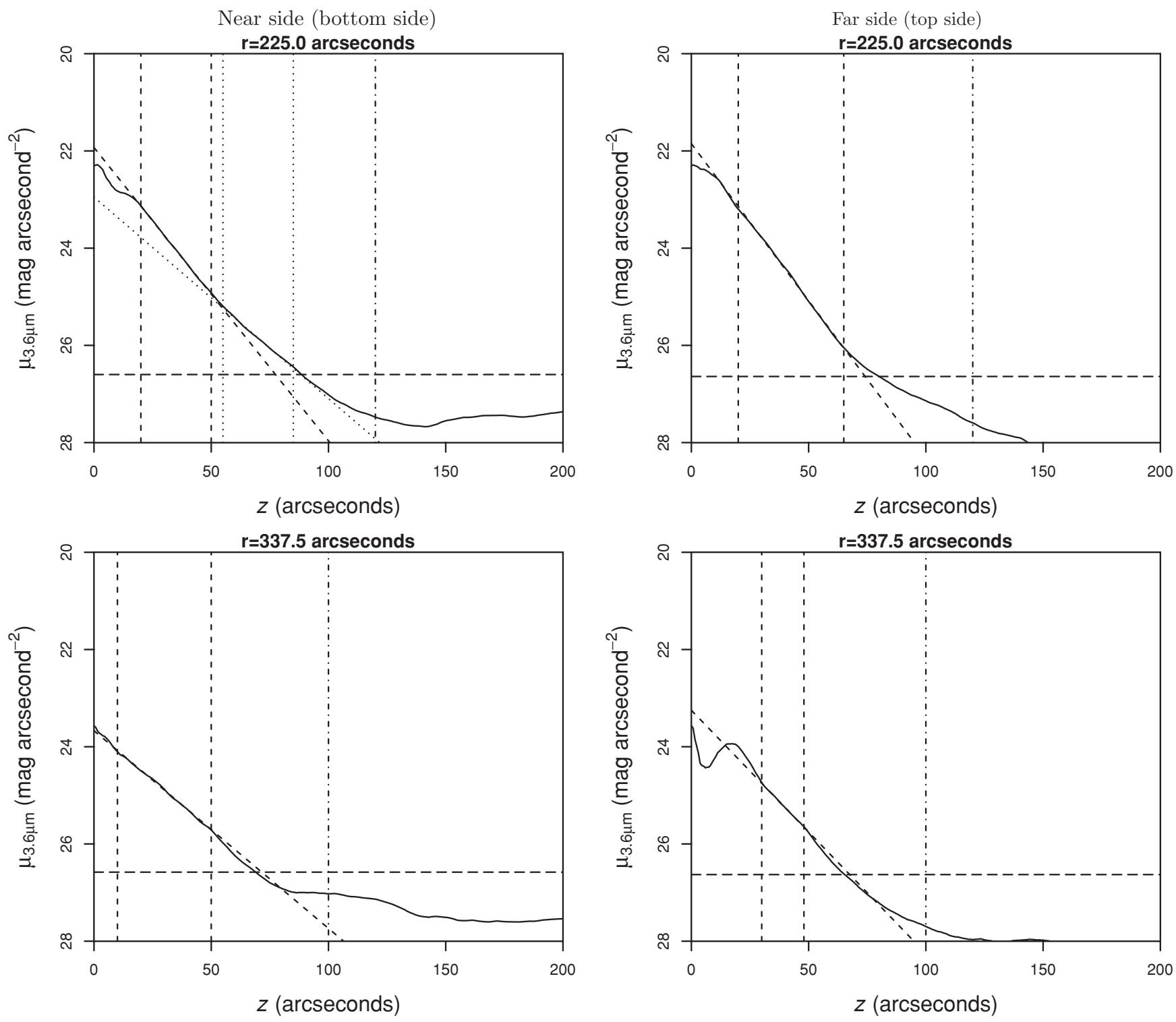

Figure 5. (Continued)

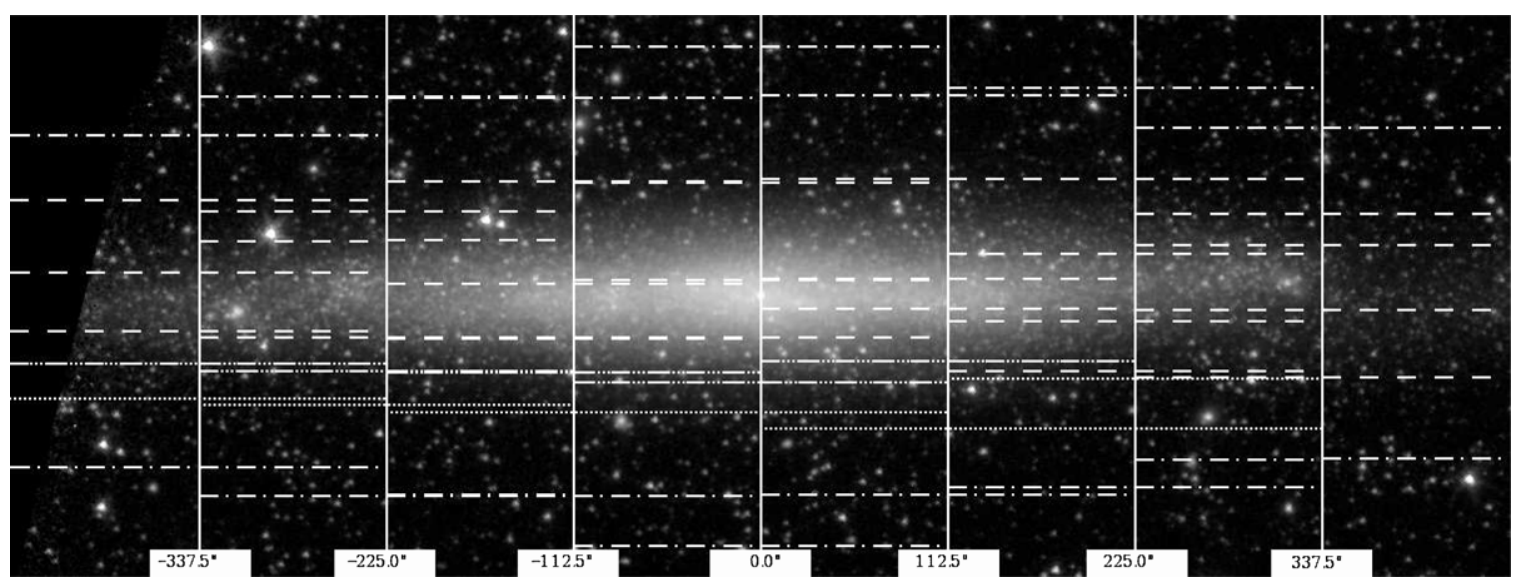

Figure 6. Representation of the fitting ranges in a $3.6 \mu \mathrm{m}$ image of NGC 4244. The displayed area is the same as in the bottom panel of Figure 1. The vertical solid lines denote the center of the bins. As in Figure 5, short dashed lines indicate the range of $z$ at which the fit to the thin disk has been done. Dotted lines indicate the range of $z$ at which the fit to the thick disk has been done. Dash-dotted lines indicate the minimum $z$ at which the noise level has been calculated. The labels at the center of each bin indicate the bin galactocentric distance, $r$. 
Table 1

Vertical Profiles Fitting Parameters and Results

\begin{tabular}{|c|c|c|c|c|c|c|c|c|}
\hline \multirow{2}{*}{$\begin{array}{l}r \\
\left({ }^{\prime \prime}\right)\end{array}$} & \multirow{2}{*}{$\begin{array}{l}z_{\mathrm{n}} \\
\left({ }^{\prime \prime}\right)\end{array}$} & \multirow{3}{*}{$\begin{array}{c}\sigma \text { Level } \\
\left(\operatorname{mag} \operatorname{arcsec}^{-2}\right) \\
(3)\end{array}$} & \multicolumn{3}{|c|}{ Inner Exponential Fit } & \multicolumn{3}{|c|}{ Outer Exponential Fit } \\
\hline & & & Range $\left({ }^{\prime \prime}\right)$ & $I_{0 \mathrm{t}}\left(\mathrm{mag} \operatorname{arcsec}^{-2}\right)$ & $H_{\mathrm{zt}}\left(^{(\prime)}\right)$ & Range $\left({ }^{\prime \prime}\right)$ & $I_{0 \mathrm{~T}}\left(\mathrm{mag} \operatorname{arcsec}^{-2}\right)$ & $H_{\mathrm{zT}}\left({ }^{\prime \prime}\right)$ \\
\hline (1) & (2) & & (4) & (5) & (6) & (7) & $(8)$ & (9) \\
\hline \multicolumn{9}{|c|}{ Far side profiles } \\
\hline-337.5 & 100 & 26.32 & $18-60$ & $22.49 \pm 0.02$ & $18.1 \pm 0.1$ & $\cdots$ & $\ldots$ & $\cdots$ \\
\hline-225.0 & 120 & 26.37 & $33-50$ & $21.18 \pm 0.02$ & $14.1 \pm 0.1$ & $\cdots$ & $\cdots$ & $\cdots$ \\
\hline-112.5 & 120 & 26.45 & $8-70$ & $20.77 \pm 0.01$ & $16.6 \pm 0.1$ & $\cdots$ & $\cdots$ & $\cdots$ \\
\hline 0.0 & 150 & 26.55 & $10-68$ & $20.33 \pm 0.01$ & $15.6 \pm 0.1$ & $\cdots$ & $\ldots$ & $\cdots$ \\
\hline 112.5 & 120 & 26.59 & $10-70$ & $20.82 \pm 0.04$ & $16.7 \pm 0.1$ & $\cdots$ & $\cdots$ & $\cdots$ \\
\hline 225.5 & 120 & 26.64 & $20-65$ & $21.85 \pm 0.03$ & $16.8 \pm 0.2$ & $\ldots$ & $\ldots$ & $\ldots$ \\
\hline 337.5 & 100 & 26.63 & $30-48$ & $23.25 \pm 0.03$ & $21.7 \pm 0.3$ & $\cdots$ & $\cdots$ & $\cdots$ \\
\hline \multicolumn{9}{|c|}{ Near side profiles } \\
\hline-337.5 & 100 & 26.45 & $18-38$ & $22.18 \pm 0.03$ & $15.1 \pm 0.2$ & $38-60$ & $22.47 \pm 0.05$ & $16.9 \pm 0.2$ \\
\hline-225.0 & 120 & 26.55 & $25-45$ & $21.25 \pm 0.03$ & $14.2 \pm 0.1$ & $45-65$ & $21.90 \pm 0.06$ & $17.5 \pm 0.3$ \\
\hline-112.5 & 120 & 26.59 & $25-45$ & $20.24 \pm 0.07$ & $13.0 \pm 0.3$ & $45-70$ & $21.22 \pm 0.05$ & $17.7 \pm 0.3$ \\
\hline 0.0 & 150 & 26.60 & $25-52$ & $20.12 \pm 0.03$ & $14.2 \pm 0.1$ & $52-70$ & $20.79 \pm 0.09$ & $17.0 \pm 0.4$ \\
\hline 112.5 & 120 & 26.60 & $8-40$ & $20.51 \pm 0.02$ & $13.9 \pm 0.1$ & $40-80$ & $21.15 \pm 0.03$ & $17.9 \pm 0.2$ \\
\hline 225.5 & 120 & 26.60 & $20-50$ & $21.93 \pm 0.02$ & $18.0 \pm 0.1$ & $55-85$ & $22.95 \pm 0.06$ & $26.2 \pm 0.5$ \\
\hline 337.5 & 100 & 26.58 & $10-50$ & $23.67 \pm 0.02$ & $26.7 \pm 0.4$ & $\ldots$ & $\ldots$ & $\ldots$ \\
\hline
\end{tabular}

Notes. The uncertainties in this table are statistical errors $(3 \sigma)$. Galactocentric distance (Column 1$)$, $z$ above which noise has been calculated (Column 2 ), noise level (Column 3), $z$ range at which the fit for the inner exponential has been calculated (Column 4), inner exponential central surface brightness (Column 5), inner exponential scale length (Column 6), $z$ range at which the fit for the outer exponential has been calculated (Column 7), outer exponential central surface brightness (Column 8), and outer exponential scale length (Column 9).

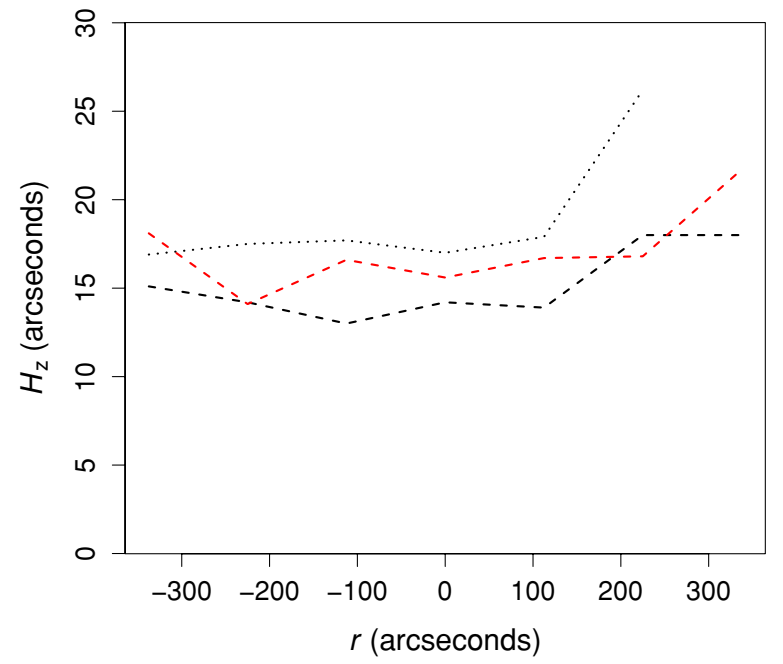

Figure 7. Scale height of the disks at different galactocentric radii. Dashed lines indicate the thin disk and the dotted line indicates the thick disk. Black lines indicate the measurements of scale heights in the near side of the galaxy and red lines the measurements in the far side of it.

(A color version of this figure is available in the online journal.)

The definition of scale height used here is different from that used in other works such as the one by Yoachim \& Dalcanton (2006) and thus the results are not directly comparable. In other papers a two-component model is fitted, but in our case, due to the very subtle change in the slope of the luminosity profile between the thin and thick disk-dominated $z$ ranges, we have considered safer to fit a single exponential in each $z$-range. As a consequence, what we have defined to be the thin disk scale height is less steep than what would be obtained from a twocomponent fit as it is influenced by the underlying thick disk component.

\subsection{Thin and Thick Disks and Opacities}

We now explore whether the fact that NGC 4244 is not totally edge-on ( $i=84.5$ according to Olling 1996) could explain the lack of traces of a thick disk in the far side of the galaxy. In other words, we want to test whether, except for the arc-like feature, the near and far sides of NGC 4244 could be intrinsically symmetric with respect to the mid-plane of the galaxy. To do so we have designed a model galaxy with two disks, each with the following light emission distribution per unit of volume (this light emission distribution can be considered as a mass distribution if we assume a constant mass-to-light ratio):

$$
\epsilon(R, z)=\epsilon_{0} e^{-R / h_{\mathrm{R}}} e^{-|z| / h_{\mathrm{z}}},
$$

where $\epsilon_{0}$ is the central emission per unit of volume, $R$ is the distance to the rotation axis of the galaxy, $z$ is the height above its mid-plane, $h_{\mathrm{R}}$ is the radial light emission distribution scale length, and $h_{z}$ is the vertical light emission distribution scale height. The parameters of each disk will be from now on designated with subindices $t$ for the thin disk and $\mathrm{T}$ for the thick disk. $\epsilon_{0}$ should not be confused with $I_{0}$, which is the fitted central surface brightness of the galaxy projected in the sky plane, and $h_{z}$ should not be confused with $H_{z}$, which is the fitted scale height of the disk in the projected galaxy. Even in the case of a galaxy with no dust and with $i=90^{\circ}, H_{z}$ would be different to $h_{z}$, as $h_{z}$ is similar to the definition of scale height used by Yoachim \& Dalcanton (2006) and $H_{z}$ is the scale height measured as a single-component exponential fit to a given range in $z$ (see the discussion at the end of Section 5.1).

The galaxy model code was designed to have a tunable thick disk central light emission, $\epsilon_{0 \mathrm{~T}}$, and a tunable thick disk mass distribution scale height $\left(h_{\mathrm{zT}}\right)$. The disk scale length was designed to be the same for the thin and the thick disk, which has been found to be true from observations as a first approximation (the ratio between thick and thin disk scale lengths is on average around 1.25 according to Yoachim \& Dalcanton 2006). We made 


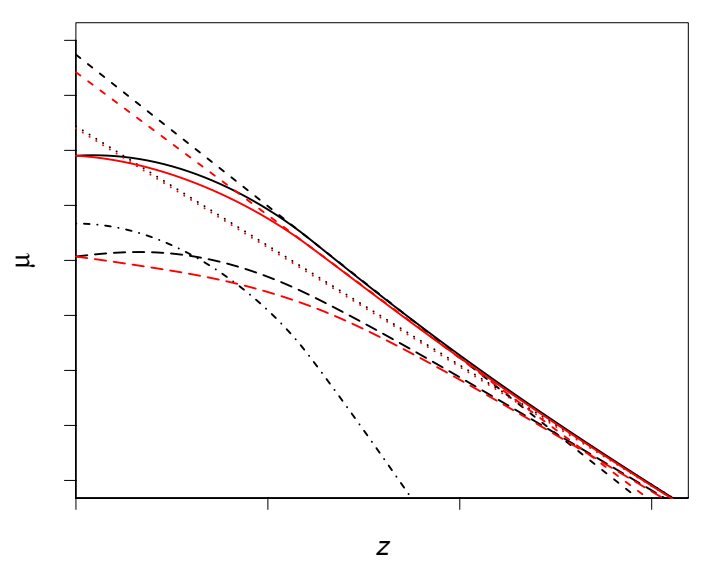

Figure 8. Representation of luminosity profiles of a highly inclined ( $i=84.5$ ) modeled galaxy with a semi-transparent thin disk with an $h_{\mathrm{zT}}=2.5 h_{\mathrm{zt}}$, $I_{0 \mathrm{~T}}=0.5 I_{0 \mathrm{t}}$, and a dust distribution in equilibrium with the thin disk component with an opacity set in a way to have an optical depth of $\tau=1$ at $z=0$ at the arbitrary galactocentric radius we are plotting. $\tau$ has been measured through the line which goes from observer eye to the infinity beyond NGC 4244 in the direction we are observing. The far side profile is shown in red and the near side profile is shown in black. Dashed lines indicate the exponential fits to the thin disk and dotted lines indicate the exponential fits to the thick disk. The dash-dotted line indicates the light contribution of the thin disk and the longdashed lines indicate the light contribution of the thick disk. The tick marks in the vertical axis are drawn every 0.5 mag. The tick marks in the horizontal axis are separated by a distance equivalent to the fitted thick disk scale length. $z=0$ has been set to be the mid-plane of the galaxy.

(A color version of this figure is available in the online journal.)

the scale height of the thin disk to be one tenth of the scale length. We made the thin disk semi-transparent, with a local density of dust proportional to the local mass density, which means that the dust is assumed to be in equilibrium with the stellar thin disk component. This assumption is the most important caveat in our modeling, as we do not know which is the real distribution of diffuse dust in NGC 4244. This implies that the results in this section are only qualitative. We assume that the thick disk is transparent, i.e., contains little or no dust. The density of dust in the thin disk is a tunable parameter in our model. Our code projects the light emitted by our three-dimensional model galaxy using a tunable inclination angle, $i$. We have studied our model at $r$ equal to one scale length of the disks.

In order to get vertical luminosity profiles similar to that in Figure 5 at the galactocentric radii at which a thick disk is detected, we have manually tuned the variable parameters of our model, except the galaxy inclination, which we have set to be $i=84.5$ (Olling 1996). We find that a good agreement is obtained when $\epsilon_{0 \mathrm{~T}}=0.5 \epsilon_{0 \mathrm{t}}, h_{\mathrm{zT}}=2.5 h_{\mathrm{zt}}$, and the dust density is set to have an optical depth $\tau=1$ when $z=0$ at the arbitrary galactocentric radius in which we are making the luminosity profile (see Figure 8 ). The selected $\tau$ is on the order of that deduced for the mid-plane of an edge-on galaxy in the $K$ band by Bianchi (2008) and thus is a reasonable upper limit for the mid-plane obscuration at $3.6 \mu \mathrm{m}$. All $\tau$ values in this paper have been measured through the line which goes from the observer to infinity, in the direction of NGC 4244. Using these parameters, we find that for the bottom parts of the galaxy the ratio between the thick and the thin disk scale height of the integrated distribution of light along the line of sight is $H_{\mathrm{zT}} \sim 1.25 \mathrm{H}_{\mathrm{zt}}$ and that the fitted central surface brightness of the thick disk is between 0.5 and 1.0 mag dimmer than that of the thin disk, which is compatible with values obtained from the fits in Figure 5.

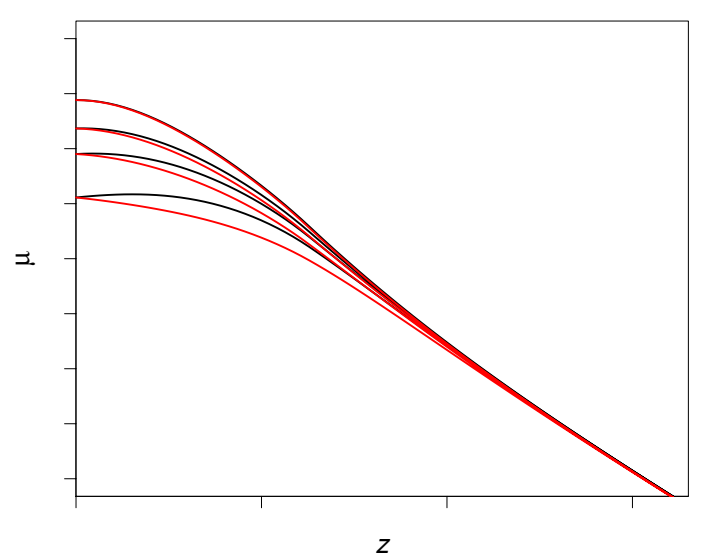

Figure 9. Representation of luminosity profiles of a highly inclined $(i=84.5)$ modeled galaxy with a semi-transparent thin disk with $h_{\mathrm{zT}}=2.5 h_{\mathrm{zt}}, I_{0 \mathrm{~T}}=$ $0.5 I_{0 \mathrm{t}}$, and a dust distribution in equilibrium with the thin disk component with an opacity set in a way to have an optical depth of $\tau=0.00, \tau=0.50, \tau=1.00$, and $\tau=2.00$ (lines from up to down) at $z=0$ and at the arbitrary galactocentric radius we are plotting. $\tau$ has been measured through the line which goes from observer eye to the infinity beyond NGC 4244 in the direction we are observing. The far side profile is shown in red and the near side profile is shown in black. The tick marks in the vertical axis are drawn every 0.5 mag. The tick marks in the horizontal axis are separated by a distance equivalent to the thick disk scale length obtained from the fit in Figure 8. $z=0$ has been set to be the mid-plane of the galaxy.

(A color version of this figure is available in the online journal.)

In Figure 5, as in our model, the luminosity profiles generally show a steeper thin disk scale height for the near side of the galaxy $(300 \pm 15 \mathrm{pc}$ against $350 \pm 30 \mathrm{pc}$ when the scale height is averaged between $r=-337^{\prime \prime} .5$ and $r=112^{\prime \prime} .5$; the uncertainty corresponds to one standard deviation of the values used for the average scale height computation). This is because for the far side of the galaxy, the thin disk opacity is obscuring the thick disk, thus removing light from the regions at low $z$, and making the slope in these regions (assigned to the thin disk in our fit) similar to that at higher $z$. This effect is seen in lines representing the thick disk luminosity profile in Figure 8.

In addition, for galactocentric radii between $r=-225^{\prime \prime} .0$ and $r=112^{\prime \prime} .5$, the arc-like feature may be hiding any trace of a softened transition from the thin to the thick disk in the far side of the galaxy. At galactocentric radii larger than $r=225^{\prime \prime}$, 0 , the galaxy disk starts to be distorted by, possibly, the presence of spiral arms, thus making the comparison between NGC 4244 and the model more difficult.

It can be argued that $\tau=1$ is a very high opacity for a galaxy imaged in $3.6 \mu \mathrm{m}$, but this opacity only corresponds to the midplane of the galaxy, where the central dust lane is found. $\tau$ drops fast with $z$, and it is on the order of $\tau=0.1$ at the $z$ at which the transition between the thin and the thick disk is found. In addition, we find that there is a degeneracy between the opacity and $\epsilon_{0 T} / \epsilon_{0 t}$. As an example, setting the mid-plane opacity as $\tau=0.6$ and $\epsilon_{0 T}=0.65 \epsilon_{0 t}$ yields a luminosity profile very similar to that in Figure 8.

We have tested, in the case of a galaxy with $i=84.5$, $\epsilon_{0 T}=0.5 \epsilon_{0 t}$, and $h_{\mathrm{zT}}=2.5 h_{\mathrm{zt}}$, the behavior of the luminosity profiles as a function of the amount of dust in the disk. The result is shown in Figure 9 in which we see that for a mid-plane optical depth of $\tau=0$, both sides are symmetric. When increasing $\tau$, the central luminosity is reduced for both near and far side profiles. The reduction in the central surface brightness causes $H_{\mathrm{zt}}$ (the measured scale height of the thin disk) to be increased, making the change in slope between the thin and the thick disk softer 


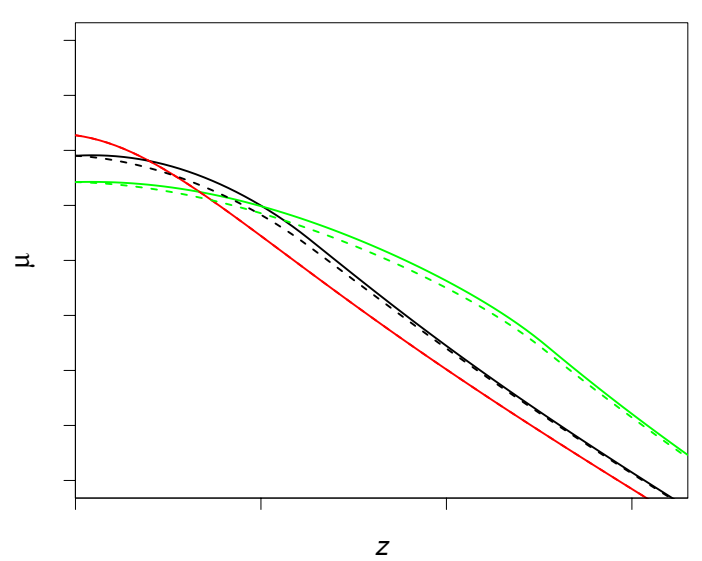

Figure 10. Representation of luminosity profiles of modeled galaxies with different inclinations $\left(i=90^{\circ} 0, i=84.5\right.$, and $i=79^{\circ} .0$ which are represented in red, black, and green, respectively) with a semi-transparent thin disk with an $h_{\mathrm{zT}}=2.5 h_{\mathrm{zt}}, I_{0 \mathrm{~T}}=0.5 I_{0 \mathrm{t}}$, and a dust distribution in equilibrium with the thin disk component with an opacity set in a way to have an optical depth of $\tau=1.00$ when $i=84.5$ at $z=0$ and at the arbitrary galactocentric radius we are plotting. $\tau$ has been measured through the line which goes from observer eye to the infinity beyond NGC 4244 in the direction we are observing. The near side profiles are shown at left and the far side profiles are shown at right. Solid lines indicate the near side and dashed lines indicate the far side. The tick marks in the vertical axis are drawn every $0.5 \mathrm{mag}$. The tick marks in the horizontal axis are separated by a distance equivalent to the thick disk scale length obtained from the fit in Figure 8. $z=0$ has been set to be the mid-plane of the galaxy.

(A color version of this figure is available in the online journal.)

with increasing $\tau$. This effect is more pronounced for the far side profile. The trend described in this paragraph is general and also applies when studying highly inclined disks with different $\epsilon_{0 T} / \epsilon_{0 t}$ and $h_{\mathrm{zT}} / h_{\mathrm{zt}}$.

We have also tested the effect of varying the galaxy inclination, $i$. The results are displayed in Figure 10. We see that for our modeled galaxy, in the cases of far side luminosity profiles the effect of a thick disk is not enhanced due to inclination effects (the ratio of scale heights between the thin and the thick disk is constant with inclination; for the model with a $\tau=1$ at $z=0$ and $i=84.5, H_{\mathrm{zT}} / H_{\mathrm{zt}}=1.20$ for $i=90.0$ and $H_{\mathrm{zT}} / H_{\mathrm{zt}}=1.20$ for $i=84.5$ ), but that in the case of near side luminosity profiles small angle differences with respect to the edge-on position make the difference between thin and thick disk scale heights larger (for the model with a $\tau=0$ at $z=0$ and $i=84.5, H_{\mathrm{zT}} / H_{\mathrm{zt}}=1.20$ for $i=90^{\prime} .0$ and $H_{\mathrm{zT}} / H_{\mathrm{zt}}=1.27$ for $i=84.5)$. Larger angle differences with respect to the edge-on position make any trace of thick disk to disappear.

Our results in this section confirm that the luminosity profiles of NGC 4244 are compatible with the galaxy having a thin and a thick disk and which are symmetric with respect to its mid-plane except for an arc-like feature on its far side.

\section{SUMMARY OF NGC 4244 FEATURES}

The features we have identified in NGC 4244 are as follows.

1. An unresolved nuclear cluster studied by Seth et al. (2008).

2. Two disk components, whose identification can only be done in the near side of the galaxy, possibly due to the presence of diffuse dust in the thin disk.

3. Possibly a spiral arm structure which causes the disk to have a slightly "warped" appearance at around 5' from the nucleus.

4. Signs of a possible tidal feature.
NGC 4244 also has an H I disk which is coplanar with the optical and near-IR one except at very large galactocentric radii $\left(r>10^{\prime}\right.$; seen in Olling 1996) where it shows a warp going in the opposite direction to the warp-like feature seen at $\sim 4^{\prime}-5^{\prime}$ in galactocentric distance.

\section{DISCUSSION AND CONCLUSIONS}

We find evidence of two disk components in a galaxy which was reported to have only one or as being a doubtful case. Using modeling we find that the fact that the thick disk is not detected in the far side of the galaxy can be explained by the effect of dust in a not perfectly edge-on galaxy.

Fry et al. (1999) did not detect the thick disk in their surface photometry because although the $R$-band image has a limiting surface brightness similar to ours, they assumed that the galaxy was symmetric with respect to the mid-plane and they summed the top and bottom luminosity profiles, which diluted the effect of the subtle thick disk. In addition, the $R$ band is more sensitive to dust, making such a subtle thick disk hard to detect in this band. Seth et al. (2005b) deduced the presence of an extended component other than the thin disk from the scale height of the oldest stars (RGB), which have a slight overdensity above the fitted sech ${ }^{-2}$ profile. However, this component is more extended than the thick disk we found and may be a halo. The sum of all stellar components they have studied (young main-sequence stars, helium-burning stars, AGB stars, and RGB stars) shows some trace of what could be a thick disk in the online color version of their Figure 3. Tikhonov \& Galazutdinova (2005) found an extended feature by counting RGB stars at the location of the arc-like feature. Seth et al. (2007) studied the RGB star distribution in a thin strip along the bottom part of the minor axis of NGC 4244 deep enough to find a halo component undetected by us due to its extreme faintness, but they did not notice any trace of the thick disk. This is probably due to the fact that the evidence for a thick disk is more subtle at $r=0^{\prime \prime}$ than at other galactocentric radii (see Figure 5). The scale height for the disk they fit $\left(\sim 300\right.$ pc or $\left.\sim 14^{\prime \prime}\right)$ is exactly the same as the one we find for the thin disk at $r=0^{\prime \prime}$.

One possible thick disk formation mechanism is dynamical heating of a thin disk. According to recent modeling (Bournaud et al. 2007, 2009), the thick disk should, at least in part, form due to gravitational instabilities. These are also responsible for the creation of giant star-forming clumps which will eventually merge to form the galaxy bulge during the first gigayear after the galaxy formation. As NGC 4244 has virtually no bulge and a thick disk which is not very differentiated from the thin disk, it is conceivable that for some reason the disk dynamical instabilities have been suppressed, which would lead to a lack of thin/thick disk differentiation. The other kind of dynamical heating is a consequence of the encounters of stars with disk substructure and is considered to be a secular evolutionary process due to thin disk irregularities such as spiral arms and giant molecular clouds, or due to dark matter subhalos. The lack of a very prominent thick disk would indicate that NGC 4244 has got a very smooth structure during most of its life. This smooth structure would have led to a modest "blurring" in the disk of the galaxy. Alternatively, NGC 4244 could have been formed much more recently than other disk galaxies studied so far.

Part of the thick disk could also have formed through accretion of stellar material from merging satellites at the time of the galaxy build-up (Yoachim \& Dalcanton 2006). According to this model the accreted gas builds the thin disk. In this formation scenario, the lack of an extended thick disk would imply that 
the merging proto-galaxies did not have a substantial stellar component at the time of the formation of NGC 4244.

Last but not the least, the thick disk may have formed in situ. According to Elmegreen \& Elmegreen (2006) edge-on galaxies with redshifts $z=0.5-4.5$ (corresponding to look-back times of 4.8-12.2 Gyr) have disk morphologies whose ratio between long and short axes is compatible with those of local thick disks. However, these disks should shrink when the galaxy accretes gaseous material, which makes the potential well deeper. In the context of this thick disk origin theory NGC 4244 may be a case of a galaxy having accreted more gaseous material than average, thus making the thick disk thinner than those in most disk galaxies. This material accretion must have been through gentle gas inflows which have settled in the thin disk.

To sum up, we have found evidence for two disk components in NGC 4244. The reason for the low degree of differentiation between the thin and the thick disk remains unknown and may be related to a lack of strong secular evolution, to a lack of stellar material in the fragments which built up the galaxy, or to a high inflow rate of gaseous material after the galaxy formation.

The authors thank the entire $S^{4} \mathrm{G}$ team for their efforts in this project. We thank our anonymous referee for giving useful advice which improved the quality of this paper. We thank Inma Martínez-Valpuesta for her useful comments. We are grateful to the dedicated staff at the Spitzer Science Center for their help and support in planning and execution of this Exploration Science program. We also gratefully acknowledge support from NASA JPL/Spitzer grant RSA 1374189 provided for the $S^{4} G$ project. K.M.D. is supported by an NSF Astronomy and Astrophysics Postdoctoral Fellowship under award AST-0802399. E.A. and A.B. thank the Centre National d'Études Spatiales for financial support.

\section{REFERENCES}

Abadi, M. G., Navarro, J. F., Steinmetz, M., \& Eke, V. R. 2003, ApJ, 597, 21 Abazajian, N. Z., et al. 2009, ApJS, 182, 534

Benson, A. J., Lacey, C. G., Frenk, C. S., Baugh, C. M., \& Cole, S. 2004, MNRAS, 351, 1215

Bergstrom, J. W., Gehrz, R. D., \& Jones, T. J. 1992, PASP, 104, 695

Bianchi, S. 2008, A\&A, 490, 461

Bournaud, F., Elmegreen, B. G., \& Elmegreen, D. M. 2007, ApJ, 670, 237
Bournaud, F., Elmegreen, B. G., \& Martig, M. 2009, ApJ, 707, L1

Brook, C. B., Kawata, D., Gibson, B. K., \& Freeman, K. C. 2004, ApJ, 612, 894 Burstein, D. 1979, ApJ, 234, 829

Dalcanton, J. J., \& Bernstein, R. A. 2002, AJ, 124, 1328

de Jong, R. S., et al. 2007, ApJ, 667, L49

Elmegreen, B. G., \& Elmegreen, D. M. 2006, ApJ, 650, 644

Fazio, G. G., et al. 2004, ApJS, 154, 10

Florido, E., Battaner, E., Sanchez-Saavedra, M. L., Prieto, M., \& Mediavilla, E. 1991, MNRAS, 251, 193

Fry, A. M., Morrison, H. L., Harding, P., \& Boroson, T. A. 1999, AJ, 118, 1209

Gilmore, G., \& Reid, N. 1983, MNRAS, 202, 1025

Hänninen, J., \& Flynn, C. 2002, MNRAS, 337, 731

Hayashi, H., \& Chiba, M. 2006, PASJ, 58, 835

Haywood, M. 2008, MNRAS, 338, 1175

Hoopes, C. G., Walterbros, R. A. M., \& Rand, R. J. 1999, ApJ, 522, 669

Kazantzidis, S., Bullock, J. S., Zentner, A. R., Kravtsov, A. V., \& Moustakas, L. A. 2008, ApJ, 688, 254

Kennicutt, R. C., Jr., Lee, J. C., Funes, S. J., José, G., Sakai, S., \& Akiyama, S. 2008, ApJS, 178, 247

Kodaira, K., \& Yamashita, T. 1996, PASJ, 48, 581

Kroupa, P. 2002, MNRAS, 330, 707

Martínez-Delgado, D., Peñarrubia, J., Gabany, R. J., Trujillo, I., Majewski, S. R., \& Pohlen, M. 2008, ApJ, 689, 184

Martínez-Delgado, D., Pohlen, M., Gabany, R. J., Majewski, S. R., \& Peñarrubia, J. 2009, ApJ, 692, 955

Olling, R. P. 1996, AJ, 112, 457

Peng, C. Y., Ho, L. C., Impey, C. D., \& Rix, H.-W. 2002, AJ, 124, 266

Peng, C. Y., Ho, L. C., Impey, C. D., \& Rix, H.-W. 2010, AJ, 139, 2097

Roskar, R., Debattista, V. P., Stinson, G. S., Quinn, T. R., Kaufmann, T., \& Wadsley, J. 2008, ApJ, 675, L65

Saha, K., de Jong, R., \& Holwerda, B. 2009, MNRAS, 396, 409

Schönrich, R., \& Binney, J. 2009a, MNRAS, 396, 203

Schönrich, R., \& Binney, J. 2009b, MNRAS, 399, 1145

Seth, A. C., Blum, R. D., Bastian, N., Caldwell, N., Debattista, V. P., \& Puzia, T. H. 2008, ApJ, 687, 997

Seth, A. C., Dalcanton, J. J., \& de Jong, R. S. 2005a, AJ, 129, 1331

Seth, A. C., Dalcanton, J. J., \& de Jong, R. S. 2005b, AJ, 130, 1574

Seth, A. C., de Jong, R., Dalcanton, J., \& GHOSTS Team 2007, in IAU Symp. Ser. 241, Stellar Populations as Building Blocks of Galaxies, ed. A. Vazdekis \& R. F. Peletier (Cambridge: Cambridge Univ. Press), 523

Shang, Z., et al. 1998, ApJ, 504, 23

Sheth, K., et al. 2010, PASP, 122, 1397

Strickland, D. K., Heckman, T. M., Colbert, E. J. M., Hoopes, C. G., \& Weaver, K. A. 2004, ApJS, 151, 193

Tikhonov, N. A., \& Galazutdinova, O. A. 2005, Astrophysics, 48, 221

Tsikoudi, V. 1979, ApJ, 234, 842

van der Kruit, P. C. 1979, A\&AS, 38, 15

van der Kruit, P. C., \& Searle, L. 1981, A\&A, 95, 105

Villumsen, J. V. 1985, ApJ, 290, 75

Yoachim, P., \& Dalcanton, J. J. 2006, AJ, 131, 226

Yoachim, P., \& Dalcanton, J. J. 2008, ApJ, 683, 707 